\title{
Performance Analysis of Three Text-Join Algorithms
}

\author{
Weiyi Meng, Member, IEEE, Clement Yu, Senior Member, IEEE, \\ Wei Wang, and Naphtali Rishe, Member, IEEE Computer Society
}

\begin{abstract}
When a multidatabase system contains textual database systems (i.e., information retrieval systems), queries against the global schema of the multidatabase system may contain a new type of joins-joins between attributes of textual type. Three algorithms for processing such a type of joins are presented and their I/O costs are analyzed in this paper. Since such a type of joins often involves document collections of very large size, it is very important to find efficient algorithms to process them. The three algorithms differ on whether the documents themselves or the inverted files on the documents are used to process the join. Our analysis and the simulation results indicate that the relative performance of these algorithms depends on the input document collections, system characteristics, and the input query. For each algorithm, the type of input document collections with which the algorithm is likely to perform well is identified. An integrated algorithm that automatically selects the best algorithm to use is also proposed.
\end{abstract}

Index Terms-Query processing, textual database, information retrieval, join algorithm, multidatabase.

\section{INTRODUCTION}

$\mathrm{R}$ ESEARCHES in multidatabase system have been intensified in recent years [4], [5], [9], [13], [12], [16], [19]. In this paper, we consider a multidatabase system that contains both local systems that manage structured data (e.g., relational DBSs) and local systems that manage unstructured data (e.g., information retrieval (IR) systems for handling text).

The global schema of a multidatabase system, integrated from local database schemas, provides an overall picture of all sharable data in the local systems. The global query language can be used to specify queries against the global schema, which will be referred to as global queries hereafter, and to retrieve data represented by the global schema. For example, if the global schema is in relational data model, then SQL can be used as the global query language. Since the multidatabase system considered in this paper contains IR components and relational components, the global query language must be capable of accommodating both structured data and unstructured data. An SQL-based query language that can serve such a purpose has been proposed in [1]. In this paper, we extend the features of this language to specify our queries.

- W. Meng is with the Department of Computer Science, State University of New York at Binghamton, Binghamton, NY 13902-6000.

E-mail:meng@cs.binghamton.edu.

- C. Yu is with the Department of Electrical Engineering and Computer Science, University of Illinois at Chicago, Chicago, IL 60607.

E-mail:yu@eecs.uic.edu.

-W. Wang is with the Computer Science Department, University of California at Los Angeles, Los Angeles, CA 90024. E-mail:weiwang@cs.ucla.edu.

- N. Rishe is with the School of Computer Science, Florida International University, Miami, FL 33199. E-mail: rishen@fiu.edu.

Manuscript received 21 Apr. 1996.

For information on obtaining reprints of this article, please send e-mail to: tkde@computer.org, and reference IEEECS Log Number 104471.
Because we have a database front-end, global users may submit queries that contain joins between attributes of textual type. A motivating example is presented in Section 2. A likely join comparator for textual attributes is SIMILAR_TO that matches objects with similar textual contents based on some similarity function. Since each textual object is essentially a document, the join is to pair similar documents among the two document collections corresponding to the two textual attributes. Although other types of comparators between textual attributes may exist, the SIMILAR_TO operator is a key operator for textual data and, therefore, we concentrate on this operator in this paper.

While processing joins between nontextual attributes has been studied extensively, not much research has been reported on processing joins between textual attributes in the literature. In [6], the authors reported a case study on automating the assignment of submitted papers to reviewers. The reported study requires matching the abstract of each submitted paper with a number of profiles of potential reviewers. The problem is essentially to process a join between two textual attributes. Since the document collections involved were small, efficient processing strategy of the join was not their concern. Instead, the emphasis of that work was on the accuracy of the automated match. A somewhat related problem is the consecutive retrieval problem [7], [17], which is to determine, for a given set of queries $Q$ against a set of records $\mathrm{R}$, whether there exists an organization of the records such that, for each query in $Q$, all relevant records (loosely, similar records) can be stored in consecutive storage locations. If we interpret $\mathrm{Q}$ and $\mathrm{R}$ as two document collections, then the consecutive retrieval problem deals with the storage aspect of efficient retrieval of relevant documents from one collection for each document from another collection. However, a major difference between consecutive retrieval problem and the join processing 
problem is that the former assumes the knowledge of which documents from $\mathrm{R}$ are relevant to each document in $\mathrm{Q}$, while the latter needs to find which documents from one collection are most similar to each document from another collection. Another related problem is the processing of a set of queries against a document collection in batch. There are several differences between this batch query problem and the join problem:

1) For the former, many statistics about the queries which are important for query processing and optimization such as the frequency of each term in the queries are not available unless they are collected explicitly, which is unlikely since the batch may only need to be processed once and it is unlikely to be cost effective to collect these statistics.

2) Special data structures commonly associated with a document collection, such as an inverted file, are unlikely to be available for the batch for the same reason given above.

As we will see in this paper, the availability of inverted files means the applicability of certain algorithms. The clustering problem in IR systems [14] requires finding, for each document $d$, those documents similar to $d$ in the same document collection. This can be considered as a special case of the join problem, as described here, when the two document collections involving the join are identical.

A straightforward way exists for processing joins between textual attributes in a multidatabase environment. This method can be described as follows: Treat each document in one collection as a query and process each such query against the other collection independently to find the most similar documents. However, this method is extremely expensive since either all documents in one of the two collections are searched or the inverted file of that collection is utilized once for processing each document in the other collection. As an example, consider the Smart system [3] developed at Cornell University. The Smart system uses inverted file to process user queries. If the collection whose documents are used as queries has a large number of documents, then using the inverted file of the other collection to process each query independently can easily incur a cost which is several orders of magnitude higher than that of a better join algorithm (see Section 6). Therefore, it is very important to develop efficient algorithms for processing joins between textual attributes. This paper has the following contributions:

1) We present and analyze three algorithms for processing joins between attributes of textual type.

2) Cost functions based on the $I / O$ cost for each of the algorithms are provided.

3) Simulation is done to compare the performance of the proposed algorithms. Our investigation indicates that no one algorithm is definitely better than all other algorithms in all circumstances. In other words, each algorithm has its unique value in different situations.

4) We provide insight on the type of input document collections with which each algorithm is likely to perform well. We further give an algorithm which determines which one of the three algorithms should be used for processing a text-join.
We are not aware of any similar study that has been reported before.

The rest of this paper is organized as follows: A motivating example is presented in Section 2. In Section 3, we include the assumptions and notations that we need in this paper. The three join algorithms are introduced in Section 4. Cost analyses and comparisons of the three algorithms are presented in Section 5. In Section 6, simulation is carried out to further compare the proposed algorithms and to suggest which algorithm to use for a particular situation. An integrated algorithm that automatically selects the best algorithm to use is also included in this section. We conclude our discussion in Section 7.

\section{A Motivating Example}

Assume that the following two global relations have been obtained after schema integration: Applicants(SSN, Name, Resume) and Positions(P\#, Title, Job_descr), where relation Applicants contains information of applicants for job positions in relation Positions, and Resume and Job_descr are of type text. Consider the query to find, for each position, $\lambda$ applicants whose resumes are most similar to the position's description. This query can be expressed in extended SQL as follows:

select P.P\#, P.Title, A.SSN, A.Name

from Positions $\mathrm{P}$, Applicants A

where A.Resume SIMILAR_TO $(\lambda)$ P.Job_descr

The where-clause of the above query contains a join on attributes of textual type. This type of joins does not appear in traditional database systems. Note that "A.Resume SIMILAR_TO $(\lambda)$ P.Job_descr" and "P.Job_descr SIMILAR_ $\mathrm{TO}(\lambda)$ A.Resume" have different semantics. The former is to find $\lambda$ resumes for each job description, while the latter is to find $\lambda$ job descriptions for each resume. All job descriptions will be listed as output by the former. However, a job description may not be listed in the output by the latter if it is not among the $\lambda$ most similar job descriptions to any resume. Later, we will see that the asymmetry of the operator SIMILAR_TO has some impact on the evaluation strategy.

There are some important differences between joins in relational database systems and the join between two textual attributes. Consider the relational join R1.A $\theta$ R2.A, where $\theta$ is a comparator such as $=$ and $>$. Given a tuple $t 1$ of R1 and a tuple $\mathrm{t} 2$ of $\mathrm{R} 2$, if $\mathrm{t} 1[\mathrm{~A}] \theta \mathrm{t} 2[\mathrm{~A}]$ is true, then we immediately know that $\mathrm{t} 1$ and $\mathrm{t} 2$ satisfy the join. However, for a given resume $r$ and a given job description $j$, there is no way for us to know immediately whether or not $r$ SIMILAR_TO $(\lambda) j$ is true, since, to be sure that $r$ is among the $\lambda$ resumes most similar to $j$, all resumes have to be considered. If we process the join by comparing each job description with all resumes, then, after a job description $d$ is compared with all resumes, the $\lambda$ resumes most similar to $d$ can be identified and a partial result is produced. However, if we process the join by comparing each resume with all job descriptions, then, after a resume is compared with all job descriptions, no partial result can be generated. In this case, many intermediate results (i.e., similarity values between resumes and job descriptions) need to be maintained 
in the main memory. This observation indicates that comparing each job description with all resumes is a more natural way to process the above textual join.

Due to selection conditions on other attributes of the relations that contain textual attributes, it is possible that only a subset of the set of documents in a collection need to participate in a join. For example, consider the query that is to find, for each position whose title contains "Engineer," • applicants whose resumes are most similar to the position's description.

\section{select P.P\#, P.Title, A.SSN, A.Name \\ from Positions P, Applicants A \\ where P.Title like "\%Engineer\%" and A.Resume SIMILAR_TO $(\lambda)$ P.Job_descr}

If selection P.Title like "\%Engineer\%" is evaluated first, then only those job descriptions whose position title contains "Engineer" need to participate in the join.

In this paper, we are interested in studying algorithms that can be used to process the following query:

select R1.X1, R2.Y2

from $R 1, R 2$

where R1.C1 SIMILAR_TO $(\lambda)$ R2.C2

where $\mathrm{C} 1$ and $\mathrm{C} 2$ are attributes representing two document collections (collection 1 and collection 2, respectively). Clearly, the join to be evaluated is of the form: " $\mathrm{C} 1$ SIMILAR_TO $(\lambda) \mathrm{C} 2$ ". The impact of selections will also be addressed.

\section{Assumptions AND NOtATIONS}

Using the vector representation [14], each document can be represented as a list of terms together with their number of occurrences in the document. Each term is associated with a weight indicating the importance of the term in the document. Usually, terms are identified by numbers to save space. We assume that each document consists of a list of cells of the form $(\mathrm{t} \#, \mathrm{w})$, called document-cell or d-cell, where $\mathrm{t} \#$ is a term number and $\mathrm{w}$ is the number of occurrences of the term $\mathrm{t}$ in the document. All d-cells in a document are ordered in ascending term numbers. The size of each $d$-cell is $|t \#|+|w|$ bytes, where $|X|$ is the number of bytes to contain $X$. In practice, $|t \#|=3$ and $|w|=2$ is sufficient. In a multidatabase environment, different numbers may be used to represent the same term in different local IR systems due to the local autonomy. Several methods may be used to overcome this problem. One method is to use actual terms rather than term numbers. The disadvantage is that the size of the document collection will become much larger. Another method is to establish a mapping between the corresponding numbers identifying the same term. Such a mapping structure, usually a table with two columns, if not stored in the main memory, can substantially degrade the performance. Assuming $|t \#|=3$, then approximately 150 pages, each of size $4 \mathrm{~KB}$, are needed for the mapping structure to accommodate 100,000 distinct terms. Since the total size of the mapping structure is less than $1 \mathrm{MB}$, it is likely that the mapping structure can be held in the memory. An attractive method is to have a standard mapping from terms to term numbers and have all local IR systems use the same mapping. Such a standard can be very beneficial in improving the performance of the multidatabase system. It can save on communication costs (no actual terms need to be transferred) and processing costs (it is more efficient to compare numbers than to compare actual terms or no need to search the mapping table). To simplify our presentation, we assume that the same number is always used to represent the same term in all local IR systems. Note that this assumption can be simulated by always keeping the mapping structure in the memory when different numbers are used to represent the same term in different local systems. In the remaining discussion, terms and term numbers will be used interchangeably.

Let $t_{1}, t_{2}, \ldots, t_{n}$ be all the common terms between documents D1 and D2. Let $u_{1}, u_{2}, \ldots, u_{n}$ and $v_{1}, v_{2}, \ldots, v_{n}$ be the numbers of occurrences of these terms in D1 and D2, respectively. The similarity between D1 and D2 can be defined as $\sum_{i=1}^{n} u_{i} * v_{i}$. A more realistic similarity function is to divide the similarity by the norms of the documents and to incorporate the use of the inverse document frequency weight [14], which assigns higher weights to terms which occur in fewer documents. The normalization can be carried out by precomputing the norms of the documents, storing them and performing the divisions during the processing of the documents. The inverse document frequency weight can be precomputed for each term and stored as parts of the list heads in the inverted files. For the sake of simplicity of presentation, we use the number of occurrences instead of weights.

For a given term $t$ in a given document collection $C$, the inverted file entry consists of a list of i-cells (short for inverted-file-cell) of the form (d\#, w), where $d \#$ is a document number and $\mathrm{w}$ is the number of occurrences of $t$ in the document with number $\mathrm{d \#}$. We assume that i-cells in each inverted file entry are ordered in ascending document numbers. The size of each i-cell is $|d \#|+|w|$. i-cells and d-cells have approximately the same size.

We use the following notations in our discussion:

$N_{i}$ - the number of documents in collection $\mathrm{i}, \mathrm{i}=1$ or 2

$\mathrm{B}$ - the size of the available memory buffer in pages

$T_{i}$ - the number of terms in collection $\mathrm{i}$

$B t_{i}$ - the size of the B+tree for collection i in pages (assume tightly packed, i.e., no space is left unused in each page except possibly the last page)

$\mathrm{p}$ - the probability that a term in collection $\mathrm{C} 1$ also appears in collection $\mathrm{C} 2$

$\mathrm{q}$ - the probability that a term in collection $\mathrm{C} 2$ also appears in collection $\mathrm{C} 1$

$\alpha$-the cost ratio of a random I/O over a sequential I/O

$\mathrm{P}$-page size in bytes (4KB)

$K_{l}$ - the average number of terms in a document in collection i

$J_{i}$ - the average size of an inverted file entry on collection $\mathrm{i}$ in pages $\left(5 *\left(K_{i} * N_{i}\right) /\left(T_{i} * P\right)\right)$

$I_{i}$ - the size of the inverted file on collection i in pages $\left(J_{i} * T_{i}\right.$, assume tightly packed)

$S_{i}$ - the average size of a document in collection $i$ in pages $\left(5 * K_{i} / P\right)$ 
$D_{i}$ - the size of collection i in pages $\left(S_{i} * N_{i}\right.$, assume tightly packed)

$I_{i}^{t}$-the inverted file entry of term $t$ on collection $\mathrm{i}$

$\lambda$-operator SIMILAR_TO $(\lambda)$ is used

$\delta$-the fraction of the similarities that are nonzero

We assume that documents in each collection are stored in consecutive storage locations. Therefore, when all documents in collection $i$ are scanned in storage order, the total number of pages read in will be $D_{i}$, which is also the total $\mathrm{I} / \mathrm{O}$ cost. On the other hand, if documents in collection $\mathrm{i}$ are read in one at a time in random order, and a document is not kept in the memory after it is processed, then the total number of pages read in will approximately be $N_{i} *\left\lceil S_{i}\right\rceil$ and the total cost will approximately be $N_{i}\left\lceil\left\lceil S_{i}\right\rceil * \alpha\right.$, where $\lceil X\rceil$ denotes the ceiling of $\mathrm{X}$ and $\alpha$ is the cost ratio of a random $\mathrm{I} / \mathrm{O}$ over a sequential $\mathrm{I} / \mathrm{O}$ due to the additional seek and rotational delay of a random read. Similarly, we assume that inverted file entries on each collection are stored in consecutive storage locations in ascending term numbers and typically $\left\lceil J_{i}\right\rceil$ pages will be read in when an inverted file entry is brought in the memory in random order.

Note that, for a given document collection, if document numbers and term numbers have the same size, then its total size is the same as the total size of its corresponding inverted file.

In this paper, only I/O cost will be used to analyze and compare different algorithms, as if we have a centralized environment where I/O cost dominates CPU cost. Cost analysis and comparisons for a distributed environment will be conducted in the future.

\section{Algorithms}

In this section, we present three algorithms for processing joins on textual attributes. These algorithms will be analyzed and compared in the next two sections. We assume the existence of the inverted file on all document collections.

Depending on how documents and/or inverted files are used to evaluate a join, three basic algorithms can be constructed. The first algorithm is to use only documents to process the join, the second algorithm is to use documents from one collection and the inverted file from another collection to evaluate the join, and the third algorithm uses inverted files from both collections to do the same job. A collection of documents can be represented by a documentterm matrix where the rows are the documents and the columns are the terms or the inverted file entries of the terms. Therefore, we name the first algorithm, the HorizontalHorizontal Nested Loop (HHNL); the second algorithm, the Horizontal-Vertical Nested Loop (HVNL); and the third, algorithm the Vertical-Vertical Merge (VVM).

\subsection{Algorithm HHNL}

A straightforward way for evaluating the join is to compare each document in one collection with every document in the other collection. Although simple, this method has several attractive properties. First, if one or two of the collections can be reduced by some selection conditions, only the remaining documents need to be considered. Second, documents can generally be read in sequentially resulting in sequential I/Os.
From the discussion in Section 2, we know that it is more natural to process the join by comparing each document in $\mathrm{C} 2$ with all documents in $\mathrm{C} 1$. That is, it is more natural to use $\mathrm{C} 2$ as the outer collection and $\mathrm{C} 1$ as the inner collection in the join evaluation. We call this order the forward order and the reverse order the backward order. The backward order can be more efficient if $\mathrm{C} 1$ is much smaller than $\mathrm{C} 2$. We consider the forward order first.

We adopt the policy of letting the outer collection use as much memory space as possible. The case that lets the inner collection use as much memory space as possible is equivalent to the backward order, which will be discussed later. With this memory allocation policy, the algorithm HHNL can be described as follows: After reading in the next $X$ documents of $C 2$ into the main memory, for some integer $\mathrm{X}$ to be determined, scan the documents in $\mathrm{C} 1$ and, while a document in $\mathrm{C} 1$ is in the memory, compute the similarity between this document and every document in $\mathrm{C} 2$ that is currently in the memory. For each document $d 2$ in $\mathrm{C} 2$, keep track of only those documents in $\mathrm{C} 1$ which have been processed against $d 2$ and have the $\lambda$ largest similarities with $d 2$.

More rigorously, with C2 as the outer collection, we need to reserve the space to accommodate at least one document in $C 1$. That is, $\left\lceil S_{1}\right\rceil$ pages of the memory need to be reserved for $\mathrm{C} 1$. We also need to reserve the space to save the $\lambda$ similarities for each document in C2 currently in the memory. Assume that each similarity value occupies 4 bytes. Then, the number of documents in $\mathrm{C} 2$ that can be held in the memory buffer of size $B$ can be estimated as: $X=(B$ $\left.-\left\lceil S_{1}\right\rceil\right) /\left(S_{2}+4 \lambda / P\right)$, where $P$ is the size of a page in bytes.

We now present the algorithm HHNL:

While (there are documents in $\mathrm{C} 2$ to be read in)

\{If there are $\mathrm{X} 1=\min \left\{N_{2}, \mathrm{X}\right\}$ or more unprocessed documents in C2 left

input the next $\mathrm{X} 1$ unprocessed documents in $\mathrm{C} 2$ into the main memory;

Else input the remaining unprocessed documents in C2 into the main memory;

For each unprocessed $d 2$ in $\mathrm{C} 2$ in the memory

For each document $d 1$ in $\mathrm{C} 1$

\{compute the similarity between $d 2$ and $d 1$;

If it is greater than the smallest of the $\lambda$ largest similarities computed so far for $d 2$

\{replace the smallest of the $\lambda$ largest similarities by the new similarity;

update the list of the documents in $\mathrm{C} 1$ to keep track of those documents with the $\lambda$ largest similarities with $d 2$;

\}

If $\lambda$ is large, then a heap structure can be used to find the smallest of the $\lambda$ largest similarities in the above algorithm.

We now consider the backward order. When $\mathrm{C} 1$ is used as the outer collection to evaluate the join, $\mathrm{C} 2$ will be scanned for each set of documents in $\mathrm{C} 1$ currently in the memory. Let $d 1$ be the first document in $\mathrm{C} 1$ read in the memory. After $\mathrm{C} 2$ is scanned, the $N_{2}$ similarities between $d 1$ 
and every document in $\mathrm{C} 2$ are computed. Since, for each document in $\mathrm{C} 2$, we need to find the $\lambda$ documents in $\mathrm{C} 1$ that are most similar to it, we need to keep track of the $\lambda$ documents in $\mathrm{C} 1$ that have the largest $\lambda$ similarities for each document in C2. This means that we need to keep track of the $\lambda * N_{2}$ similarities during the backward order evaluation. In other words, we need a memory space of size $4 \lambda * N_{2} / P$ to keep these similarities. Compared with the forward order which requires $4 \lambda * X 1 / P$ pages to keep track of the needed similarities, more memory space is needed to save the similarities for the backward order. This will have an adverse impact on the performance of the backward order. As a result, the forward order is likely to perform better than the backward order when the two document collections have about the same size. However, when $\mathrm{C} 1$ is much smaller than $\mathrm{C} 2$, then the backward order can still outperform the forward order. For example, if $\mathrm{C} 1$ can be entirely held in the memory, then only one scan of each collection is needed to process the join with the backward order no matter how large $\mathrm{C} 2$ is.

\subsection{Algorithm HVNL}

This algorithm uses the documents in one collection and the inverted file for the other collection to compute the similarities. In an information retrieval system, processing a user query, which can be considered as a document, is to find the $\lambda$ documents in the system which are most similar to the user query. One way to process such a query is to compare it with each document in the system. This method requires almost all nonzero entries in the document-term matrix be accessed. A more efficient way is to use the inverted file on the document collection to process the query. This method is used in the Smart system [3]. The advantage of this method is that it only needs to access those nonzero entries in the columns of the document-term matrix which correspond to the terms in the query. Since the number of terms in a query is usually a very small fraction of the total number of terms in all documents in the system, the inverted file based method accesses only a very small portion of the document-term matrix. Algorithm HVNL is a straightforward extension of this method to the situation where we need to find the $\lambda$ most similar documents from one collection for every document in another collection.

The process of using the inverted file to compute the similarities between a document $d$ in $\mathrm{C} 2$ to documents in $\mathrm{C} 1$ can be described as follows. Let $(t, w)$ be the next d-cell to be considered in $d$. Let the inverted file entry corresponding to $t$ on $\mathrm{C} 1$ be $\left\{\left(d_{1}, w_{1}\right), \ldots,\left(d_{n}, w_{n}\right)\right\}$, where $d_{i}$ s are document numbers. After $t$ is processed, the similarity between $d$ and document $d_{i}$ as accumulated so far will be $U_{i}+w * w_{i}$, where $U_{i}$ is the accumulated similarity between $d$ and $d_{i}$ before $t$ is considered, and $w * w_{i}$ is the contribution due to the sharing of the term $t$ between $d$ and $d_{i}, \mathrm{i}=1, \ldots, \mathrm{n}$. After all terms in $d$ are processed, the similarities between $d$ and all documents in $\mathrm{C} 1$ will be computed, and the $\lambda$ documents in $\mathrm{C} 1$ which are most similar to $d$ can be identified.

Note that before the last $\mathrm{d}$-cell in $d$ is processed, all intermediate similarities between $d$ and all documents in $\mathrm{C} 1$ need to be saved. The amount of memory needed for such purpose is proportional to $N_{1}$. Further analysis can reveal that using the inverted file on C2 to process the join needs more memory space to store intermediate similarities (the amount is proportional to $\lambda * N_{2}$ ). In practice, only nonzero similarities need to be saved. We use $\delta$ to denote the fraction of the similarities that are nonzero, $0<\delta<1$.

A straightforward way to process the join is to go through the above process for each document in C2 independently. That is, read in each document $d$ in C2 in turn and, while $d$ is in the memory, read in all inverted file entries on $\mathrm{C} 1$ corresponding to terms in $d$ to process $d$ (note that not all terms in $d$ will necessarily appear in $\mathrm{C} 1$ ). The problem with this straightforward method is its lack of coordination between the processing of different documents in C2. As a result, if a term appears in K documents in C2, then the inverted file entry of the term (assume that it also appears in C1) on C1 will be read in K times. Algorithm HVNL is designed to reuse the inverted file entries that are read in the memory for processing earlier documents to process later documents to save I/O cost. Due to space limitation, usually not all inverted file entries read in earlier can be kept in the memory. Therefore, the algorithm also needs a policy for replacing an inverted file entry in the memory by a new inverted file entry. Let the frequency of a term in a collection be the number of documents containing the term. This is known as document frequency. Document frequencies are stored for similarity computation in IR systems and no extra effort is needed to get them. Our replacement policy chooses the inverted file entry whose corresponding term has the lowest frequency in C2 to replace. This reduces the possibility of the replaced inverted file entry to be reused in the future. To make the best use of the inverted file entries currently in the memory, when a new document $d 1$ in $\mathrm{C} 2$ is processed, terms in $d 1$ whose corresponding inverted file entries are already in the memory are considered first. This means that each newly read in document will be scanned twice in the memory. The first scan is to find the terms whose corresponding inverted file entries are already in the memory and the second scan is to process other terms. A list that contains the terms whose corresponding inverted file entries are in the memory will be maintained. Note that, when not all inverted file entries that are read in earlier can be kept in the memory, it is still possible to read in an inverted file entry more than one time. Note also that the worst case scenario for algorithm HVNL is that for each document in C2 under consideration, none of its corresponding inverted file entries is currently in the memory. In this case, algorithm HVNL deteriorates into the straightforward method.

We now present the algorithm HVNL:

For each document $d$ in C2

\{For each term $t$ in $d$

If $t$ also appears in $\mathrm{C} 1$

If the inverted file entry of $t$ on $\mathrm{C} 1\left(I_{1}^{t}\right)$ is in the memory accumulate similarities;

For each term $t$ in $d$

If $t$ also appears in $\mathrm{C} 1$

\{If the inverted file entry of $t$ on $\mathrm{C} 1\left(I_{1}^{t}\right)$ is not in the memory 
If the available memory space can accommodate $I_{1}^{t}$ read in $I_{1}^{t}$; Else

find the inverted file entry in the memory with the lowest document frequency and replace it with $I_{1}^{t}$;

accumulate similarities;

\}

find the documents in $\mathrm{C} 1$ which have the $\lambda$ largest similarities with $d$; \}

For each inverted file, there is a B+tree which is used to find whether a term is in the collection and if present where the corresponding inverted file entry is located.

One possible way to improve the above algorithm is to improve the selection of the next document to process. Intuitively, if we always choose an unprocessed document in C2 whose terms' corresponding inverted file entries on C1 have the largest intersection with those inverted file entries already in the memory as the next document to process, then the likelihood of an inverted file entry already in the memory to be reused can be increased. For example, consider three documents each with three terms: D1 $=\left\{t_{1}, t_{2}, t_{3}\right\}$, D2 $=\left\{t_{2}, t_{3}, t_{4}\right\}$, and D3 $=\left\{t_{3}, t_{4}, t_{5}\right\}$. Suppose terms with smaller subscripts have lower document frequencies. Suppose the memory buffer is only large enough to hold three inverted file entries. If D1, D2, and D3 are processed in the given order, then each inverted file entry needs to be read in exactly once. However, if the processing order is D1, D3, and D2, then the inverted file entry corresponding to t2 will be read in twice and all other inverted file entries will be read in exactly once. Clearly, for this example, order $\{\mathrm{D} 1, \mathrm{D} 2, \mathrm{D} 3\}$ is better than order $\{\mathrm{D} 1, \mathrm{D} 3, \mathrm{D} 2\}$.

An order is optimal if it incurs the minimum $\mathrm{I} / \mathrm{O}$ cost. The question is can an optimal order be found efficiently. Unfortunately, as shown by the proposition below, the problem of finding an optimal order is NP-hard.

PROPOSITION. The problem of finding an optimal order of documents in C2 so that the best performance can be achieved is NP-hard.

PROOF. It was shown in [11] that the following problem, known as the Optimal Batch Integrity Assertion Verification (OBIAV), which is to find an optimal order for verifying a set of integrity constraints and verifying each such constraint requires a set of pages be brought in from secondary storage to the memory, is an NP-hard problem. It can be seen that the optimal order problem in our case is essentially the same as the optimal order problem in OBIAV because the following correspondences between the two problems can be easily established: Processing a document in C2 corresponds to verifying an integrity constraint; the need to read in a set of inverted file entries for processing each document in C2 corresponds to the need to bring in a set of pages for verifying each integrity constraint; that an inverted file entry read in for processing one document may be used for processing another document corresponds to that a page brought in for verifying one integrity constraint may be used for verifying another integrity constraint. Therefore, the optimal order problem in our case is also NP-hard.

We decided not to pursue the issue of finding an optimal order further because in addition to its NP-hard nature, there is another problem associated with any optimal order, that is, by reading in documents in any order rather than their storage order, more expensive random I/Os will be incurred.

\subsection{Algorithm VVM}

Algorithm VVM uses inverted files on both collections to compute the similarities. The strength of this algorithm is that it only needs to scan each inverted file once to compute similarities between every pair of documents in the two collections regardless of the sizes of the two collections provided that the memory space is large enough to accommodate intermediate similarity values. In this case, algorithm VVM can be at least as good as algorithm HHNL because algorithm HHNL needs to scan each document collection at least once and the size of the inverted file on a collection is about the same as the size of the collection itself. Algorithm VVM tries to compute similarities between every pair of documents in the two collections simultaneously, as a result, it needs to save the intermediate similarities. Thus, the memory requirement for saving these similarities is proportional to $N_{1} * N_{2}$ (independent of the number of terms in each document), which can be so large such that algorithm VVM cannot be run at all. In summary, algorithm VVM is likely to perform well for document collections that are large in size (such that none can be entirely held in the memory) but small in number of documents. This is possible if each document has a large size. Another situation that algorithm VVM may do well is when the vocabularies of the two document collections are very different. For example, one collection is on medicine and the other is on computer science. In this case, the number of nonzero similarities between documents in the two collections is likely to be small.

Algorithm VVM can be described as follows: We scan both inverted files on the two collections. During the parallel scan, if two inverted file entries correspond to the same term, then invoke the similarity accumulating process.

Recall that we assumed that inverted file entries are stored in ascending term numbers. Therefore, one scan of each inverted file is sufficient (very much like the merge phase of merge sort). The similarity accumulating process can be described as follows. Let $I_{1}^{t}=\left\{\left(r_{1}, u_{1}\right), \ldots,\left(r_{m}, u_{m}\right)\right\}$ and $I_{2}^{t}=\left\{\left(s_{1}, v_{1}\right), \ldots,\left(s_{n}, v_{n}\right)\right\}$ be two inverted file entries for the same term $t$ on the two collections, respectively. After the two inverted file entries are processed, the similarity between documents $r_{p}$ and $s_{q}$ as accumulated so far will be $U_{p q}+u_{p} * v_{q}$, where $U_{p q}$ is the accumulated similarity between $r_{p}$ and $s_{q}$ before $t$ is considered, $p=1, \ldots, m, q=1, \ldots, n$.

We can extend the above algorithm VVM as follows to tackle the problem of insufficient memory space for all intermediate similarities. Suppose SM is the total number of 
pages needed to store the intermediate similarities when all pairs of documents in the two collections are considered at the same time. Suppose $M$ is the available memory space for storing the intermediate similarities. If $\mathrm{SM}>\mathrm{M}$, divide collection C2 into $\lceil S M / M\rceil$ subcollections and then compute the similarities between documents in each subcollection and documents in $\mathrm{C} 1$, one subcollection at a time. Since, for each such subcollection, one scan of the original inverted files on both collections is needed, this extension incurs a cost which will be $\lceil S M / M\rceil$ times higher than that when the memory is large enough to hold all intermediate similarities. For a more detailed cost analysis, see Section 5.3.

\section{I/O Cost Analysis}

In this section, we provide analysis of the $\mathrm{I} / \mathrm{O}$ cost of each algorithm presented in Section 4.

\subsection{Algorithm HHNL}

Let $X$ be the number of documents in $C 2$ that can be held in the memory buffer of size B, as defined in Section 4.1. Since, for each $\mathrm{X}$ documents in $\mathrm{C} 2, \mathrm{C} 1$ needs to be scanned once, the total I/O cost of HHNL can be estimated as below:

$$
\text { hhs }=D_{2}+\left\lceil N_{2} / X\right\rceil * D_{1}
$$

(HHS1),

where the first term is the cost of scanning $\mathrm{C} 2$ and the second term is the cost of scanning $\mathrm{C} 1$, and $\left\lceil N_{2} / X\right\rceil$ is the number of times $C 1$ needs to be scanned.

The above cost formula assumes that all I/Os are sequential I/Os (i.e., both $\mathrm{C} 1$ and $\mathrm{C} 2$ are sequentially scanned in). This is reasonable only when each document collection is read by a dedicated drive with no or little interference from other $\mathrm{I} / \mathrm{O}$ requests. If this is not the case, then some of the I/Os may become more costly random I/Os. We first consider the case when $N_{2} \geq X$. The following interleaved $\mathrm{I} / \mathrm{O}$ and $\mathrm{CPU}$ patterns can be observed. After each $X$ documents in $\mathrm{C} 2$ are read in, for each document $d$ in $\mathrm{C} 1$ read in, the CPU will take some time to compute the similarities between the $\mathrm{X}$ documents and $d$. When the CPU is doing the computation, $\mathrm{I} / \mathrm{O}$ resources may be allocated to other jobs. If this is the case, then the next document from $\mathrm{C} 1$ will use a random $\mathrm{I} / \mathrm{O}$, so does the read-in of the next $\mathrm{X}$ documents in C2. In other words, in the worst case, all documents in $\mathrm{C} 1$ will be read in using random $\mathrm{I} / \mathrm{O}$ and for every $X$ documents in $C 2$, there will be a random $\mathrm{I} / \mathrm{O}$. The number of actual random I/Os for scanning documents in C1 once also depends on the document size and can be estimated as $\min \left\{D_{1}, N_{1}\right\}$ (if $S_{1} \leq 1$, then $D_{1}$ should be used; otherwise, $N_{1}$ should be used). Therefore, when $N_{2} \geq X$, in the worst scenario, the total I/O cost can be estimated as follows:

$$
h h r=h h s+\left\lceil N_{2} / X\right\rceil *\left(1+\min \left\{D_{1}, N_{1}\right\}\right) *(\alpha-1) .
$$

When $N_{2}<X$, then the entire collection C2 can be scanned in sequentially and held in the memory, and the remaining memory space $\left(\left(X-N_{2}\right) * S_{2}\right)$ can be used to hold documents in $\mathrm{C} 1$. Therefore, $\mathrm{C} 1$ can be read in $\left\lceil D_{1} /((X\right.$ $\left.\left.\left.-N_{2}\right) * S_{2}\right)\right\rceil$ blocks and each block can be read in sequentially. In this case, we have

$$
h h r=h h s+\left\lceil D_{1} /\left(\left(X-N_{2}\right) * S_{2}\right)\right\rceil *(\alpha-1) .
$$

\subsection{Algorithm HVNL}

Recall that a B+tree is maintained for each document collection for quickly locating the inverted file entry of any given term. The size of the $\mathrm{B}+$ tree can be estimated as follows: Typically, each cell in the B+tree occupies 9 bytes ( 3 for each term number, 4 for address, and 2 for document frequency). If a document collection has $\mathrm{N}$ terms, then the size of the B+tree is approximately $9 * \mathrm{~N} / \mathrm{P}$ (only the leaf nodes are considered). The size is not terribly large. For example, for a document collection with 100,000 distinct terms, the $\mathrm{B}+$ tree takes about 220 pages of size $4 \mathrm{~KB}$. We assume that the entire B+tree will be read in the memory when the inverted file needs to be accessed and it incurs a one-time cost of reading in the B+tree.

Let $X$ be the number of inverted file entries on $C 1$ that can be held in the memory when the memory buffer is fully used. In addition to $X$ inverted file entries, the memory (size B) also needs to contain a document in $\mathrm{C} 2$ of size $\left\lceil S_{2}\right\rceil$, a $\mathrm{B}+$ tree of size $B t_{1}$, the nonzero similarities values between the document in C2 currently under processing and all documents in $\mathrm{C} 1$ and the list containing the terms whose corresponding inverted file entries are in the main memory (size $X|t \#| / P$ ). Therefore, $X$ can be estimated as follows:

$$
X=\left\lfloor\frac{B-\left\lceil S_{2}\right\rceil-B t_{1}-4 * N_{1} \delta / P}{J_{1}+|t \#| / P}\right\rfloor .
$$

If we assume that the read-in of the documents in $\mathrm{C} 2$ incurs sequential I/Os, then the I/O cost of HVNL can be estimated as follows:

$$
\begin{gathered}
\text { hvs }= \\
\begin{cases}\min \left\{D_{2}+I_{1}+B t_{1}, D_{2}+T_{2} * q *\left\lceil J_{1}\right\rceil *\right. & \\
\left.\alpha+B t_{1}\right\} & \text { if } X \geq T_{1} \\
D_{2}+T_{2} * q *\left\lceil J_{1}\right\rceil * \alpha+B t_{1}, & \text { if } T_{1}>X \geq T_{2} * q \\
D_{2}+X *\left\lceil J_{1}\right\rceil * \alpha+\left(N_{2}-s-X 1+1\right) * & \\
Y *\left\lceil J_{1}\right\rceil * \alpha+B t_{1} & \text { otherwise }\end{cases}
\end{gathered}
$$

where the first case corresponds to the case when $X$ is greater than or equal to the total number of inverted file entries on $\mathrm{C} 1$ (i.e., $T_{1}$ ). In this case, we can either read in the entire inverted file on $\mathrm{C} 1$ in sequential order (this corresponds to the first expression in $\min \{\})$ or read in all inverted file entries needed to process the query (the number is $T_{2} * q$ ) in random order. (This corresponds to the second expression in $\min \{\}$. The memory is large enough to do this, since $X \geq T_{1} \geq T_{2} * q$.) The second case corresponds to the case when the memory is not large enough to hold all inverted file entries on $\mathrm{C} 1$ but is large enough to hold all of the necessary inverted file entries; the last expression is for the case when the memory is not large enough to hold all needed inverted file entries on $\mathrm{C} 1$. In this case, the second term is the cost of finding and reading in the inverted file entries on $\mathrm{C} 1$ which correspond to the terms in documents in C2 until the memory is fully occupied. Suppose the memory is just large enough to hold all the inverted file entries on $\mathrm{C} 1$ corresponding to the terms in the first $(s-1)$ documents in C2 and a fraction (X1) of the inverted file entries corresponding to the terms in the sth document in C2 
(i.e., the inverted file entries on $\mathrm{C} 1$ corresponding to the terms in the first $s+X 1-1$ documents in $\mathrm{C} 2$ can be held in the memory). Let $Y$ be the number of new inverted file entries that need to be read in when a new document in $\mathrm{C} 2$ is processed after the memory is fully occupied. Then, the third term is the total cost of reading in new inverted file entries for processing the remaining documents in $\mathrm{C} 2$. We now discuss how $s, \mathrm{X} 1$, and $\mathrm{Y}$ can be estimated. First, the number of distinct terms in $m$ documents in $\mathrm{C} 2$ can be estimated by $f(m)=T_{2}-\left(1-K_{2} / T_{2}\right)^{m} * T_{2}$. Therefore, $s$ is the smallest $m$ satisfying $q * f(m)>X$. Note that $(X-q * f(s-1))$ is the number of inverted file entries that can still be held in the memory after all the inverted file entries on $\mathrm{C} 1$ corresponding to the terms in the first $(s-1)$ documents in $\mathrm{C} 2$ have been read in and $(q * f(s)-q * f(s-1))$ is the number of new inverted file entries that need to be read in when the sth document in $\mathrm{C} 2$ is processed, $\mathrm{X} 1$ can be estimated by $(X-q$ $* f(s-1)) /(q * f(s)-q * f(s-1))$. Finally, $\mathrm{Y}$ can be estimated by $(q * f(s+X 1)-X)$.

As discussed in Section 5.1, it is possible that some or all of the I/Os of reading in the documents in $\mathrm{C} 2$ are random $\mathrm{I} / \mathrm{Os}$ due to other obligations of the I/O device. If, after inverted file entries are accommodated, there is still more memory space left, then the remaining memory space can be used to sequentially scan in multiple documents in $\mathrm{C} 2$ at a time. Based on this observation, when random I/Os are considered, the total I/O cost of HVNL can be estimated as:

$$
\begin{aligned}
& h v r= \\
& \int \min \left\{D_{2}+I_{1}+B t_{1}+\left\lceil D_{2} /\left(\left(X-T_{1}\right) * J_{1}\right)\right\rceil\right. \\
& *(\alpha-1), D_{2}+T_{2} * q *\left\lceil J_{1}\right\rceil * \alpha+B t_{1} \quad \text { if } X \geq T_{1} \\
& \left.+\left\lceil D_{2} /\left(\left(X-T_{2} * q\right) * J_{1}\right) *(\alpha-1)\right\rceil\right\} \\
& \text { hvs }+\left[D_{2} /\left(\left(X-T_{2} * q\right) * J_{1}\right)\right] *(\alpha-1), \quad \text { if } T_{1}>X \geq T_{2} * q \\
& h v s+\min \left\{D_{2}, N_{2}\right\} *(\alpha-1) \\
& \text { otherwise. }
\end{aligned}
$$

It would be easier to understand the above formula when compared with the formula for computing hos. In the first expression in $\min \{\},\left(X-T_{1}\right) * J_{1}$ is the remaining memory space after all inverted file entries are accommodated.

With slight modification on similarity accumulation, C1 can be used as the outer collection to process the query. In this case, the memory space needed to store intermediate similarities will be $4 \lambda \delta N_{2} / P$. The cost of the backward order can be estimated in the same way as in the case of the forward order.

\subsection{Algorithm VVM}

To avoid the much higher cost of random I/Os, we can simply scan both inverted files on the two collections. During the parallel scan, if two inverted file entries correspond to the same term, then invoke the similarity accumulating process. Recall that we assumed the inverted file entries are stored in ascending term numbers. Therefore, one scan of each inverted file is sufficient to compute all similarities if the memory is large enough to accommodate all intermediate similarities. Therefore, if all the I/Os are sequential I/Os, the total I/O cost of the algorithm VVM is:

$$
v v s=I_{1}+I_{2} \text {. }
$$

Again, some or all of the I/Os could actually be random $\mathrm{I} / \mathrm{Os}$ due to other obligations of the $\mathrm{I} / \mathrm{O}$ device. In the worst case scenario, i.e., all I/Os are random I/Os, the total I/O cost of the algorithm VVM can be estimated as:

$$
\text { vvr }=\left(\min \left\{I_{1}, T_{1}\right\}+\min \left\{I_{2}, T_{2}\right\}\right) * \alpha .
$$

Algorithm VVM usually requires a very large memory space to save the intermediate similarity values. If only nonzero similarities are stored, then the memory space for storing intermediate similarity values for the algorithm VVM is $4 \delta * N_{1} * N_{2} / P$. When the memory space is not large enough to accommodate all intermediate similarity values, a simple extension to the algorithm VVM can be made (see Section 4.3). In this case, the total cost can be estimated by multiplying vos (or $v v r$ ) by $\lceil S M / M\rceil$, where $S M=4 \delta * N_{1}$ * $\mathrm{N}_{2} / P$ is the total number of pages needed to store the intermediate similarities when all pairs of documents in the two collections are considered at the same time and $\mathrm{M}=B-$ $\left\lceil J_{1}\right\rceil-\left\lceil J_{2}\right\rceil$ is the available memory space for storing the intermediate similarities. Therefore, a more general formula for estimating the total I/O cost when all the I/Os are sequential I/Os can be given below:

$$
\text { vvs }=\left(I_{1}+I_{2}\right) *\lceil S M / M\rceil
$$

and a more general formula for estimating the total $\mathrm{I} / \mathrm{O}$ cost when all the I/Os are random I/Os is:

$$
\text { vor }=\left(\min \left\{I_{1}, T_{1}\right\}+\min \left\{I_{2}, T_{2}\right\}\right) * \alpha *\lceil S M / M\rceil .
$$

\subsection{Comparisons}

Algorithm HHNL uses two document collections as the input. Each of the two document collections needs to be scanned at least once, which constitutes the lower bound of the I/O cost of this algorithm. Algorithm HHNL does not use any special data structures, such as inverted files and $\mathrm{B}+$ trees. Thus, it is more easily applicable and easier to implement. Since algorithm HHNL uses documents directly for similarity computation, it benefits quite naturally from any possible reductions to the number of documents in either one or both collections resulted from the evaluation of selection conditions on nontextual attributes of the relevant relations. The memory space requirement of this algorithm for storing intermediate similarity values is generally small compared with those of other algorithms.

Algorithm HVNL uses one document collection, one inverted file, and the B+tree corresponding to the inner collection as the input. While the document collection is always scanned once, the access to inverted file entries is more complex. On the one hand, not all inverted file entries need to be read in. In fact, only those inverted file entries whose corresponding terms also appear in the other document collection need to be accessed. On the other hand, some inverted file entries may be read in many times due to their appearances in multiple documents in $\mathrm{C} 2$, although effort is made by the algorithm to reuse inverted file entries currently in the memory. It is expected that this algorithm can be very competitive in the following two situations:

1) One of the document collection, say $C 2$, is much smaller than the other collection. In this case, it is likely that only a small fraction of all inverted file en- 
tries in the inverted file needs to be accessed. This means that only a small portion of the document-term matrix corresponding to $\mathrm{C} 1$ will be accessed in this case. In contrast, if algorithm HHNL is used, then the entire matrix needs to be accessed at least once, even when $\mathrm{C} 2$ can be held entirely in the memory.

When $\mathrm{C} 2$ contains only one document, this situation becomes an extreme case of processing a single query against a document collection. As we have mentioned before, using the inverted file to process a single query has been shown in IR to be superior to using documents directly. Note that an originally large document collection may become small after conditions on attributes of the relevant relation are evaluated.

2) For the collection where documents are used, close documents in storage order share many terms and nonclose documents share few terms. This increases the possibility of reusing inverted file entries in the memory and reduces the possibility of rereading in inverted file entries. This could happen when the documents in the collection are clustered.

Algorithm HVNL accesses inverted file entries in random order. As such, it has two negative effects on the $\mathrm{I} / \mathrm{O}$ cost. One is that random I/Os are more expensive than sequential I/Os. The other is that, even when an inverted file entry occupies a small fraction of a page, the whole page containing the entry has to be read in. In other words, if $e$ is the size of an inverted file entry, we need to read in $\lceil e\rceil$ even if $e$ is very small, say 0.1 . Therefore, when the size of each inverted file entry is close to an integer, the competitiveness of algorithm HVNL will be increased. Algorithm HVNL uses primarily two data structures, one is the inverted file and the other is the B+tree for the terms. One disadvantage of using the inverted file is that the size of the file remains the same even if the number of documents in the corresponding document collection can be reduced by a selection unless we construct another inverted file for the reduced set, which is highly unlikely due to the cost involved. The memory space requirement of algorithm HVNL for storing intermediate similarities is higher than that of algorithm HHNL but lower than that of algorithm VVM.

Algorithm VVM uses two inverted files as the input. As we discussed before, this algorithm has a very nice one-scan property, namely, it only needs to scan each inverted file once to compute the similarities regardless of the sizes of the two collections provided that the memory space is large enough to accommodate intermediate similarity values. When the memory space is large enough to accommodate intermediate similarity values, algorithm VVM can be at least as efficient as algorithm HHNL as far as I/O cost is concerned. The major drawback of algorithm VVM is that it needs a very large memory space to save the intermediate similarities. There are two situations in which algorithm VVM is likely to perform well. The first is when the document collections are large in size but small in number of documents. The second is when the vocabularies of the two document collections are very different. In both of the two situations, the number of nonzero similarities between documents in the two collections is likely to be small. Another disadvantage of algorithm VVM is that the sizes of the inverted files will remain the
TABLE 1

STATISTICAL INFORMATION of SEVERAL DOCUMENT COLLECTIONS

\begin{tabular}{|l|c|c|c|}
\hline \hline & WSJ & FR & DOE \\
\hline \#documents & 98,736 & 26,207 & 226,087 \\
\#terms per doc & 329 & 1,017 & 89 \\
total \# of distinct terms & 156,298 & 126,258 & 186,225 \\
collection size in pages & 40,605 & 33,315 & 25,152 \\
avg. size of a document & 0.41 & 1.27 & 0.111 \\
avg. size of an inv. fi. en. & 0.26 & 0.264 & 0.135 \\
\hline
\end{tabular}

same even if the number of documents in the corresponding document collections can be reduced.

\section{Simulation Results}

Due to the large number of parameters in the cost formulas of the algorithms presented, it is very difficult to compare the performance of these algorithms based on these formulas directly. In this section, the algorithms are compared based on simulation results computed from the cost formulas derived in Section 5. Our objective is to identify the impact of the variations of the parameters on the algorithms. In other words, we would like to find out in what situation an algorithm performs the best.

The statistics of three document collections which were collected by ARPA/NIST [8], namely, WSJ (the Wall Street Journal), FR (Federal Register), and DOE (Department of Energy), are used in our simulation. The statistics of these collections are shown in Table 1 (the last three rows are estimated by us based on $|t \#|=3$ ).

Among the three document collections, FR has fewer, but larger, documents and DOE has more, but smaller, documents. The number of documents in WSJ lies between those of FR and DOE. So is the average size of documents in WSJ.

For all simulations, the page size $P$ is fixed at $4 \mathrm{~KB}$, the fraction of the similarities that are nonzero $\delta$ is fixed at 0.1 , and $\lambda$ is fixed at 20 (note that only algorithm HHNL and the backward order of algorithm HVNL involve $\lambda$ and none is really sensitive to $\lambda$ if it is not very large, say in the hundreds). The probability $q$ is computed as follows:

$$
q= \begin{cases}0.8 * T_{1} / T_{2}, & \text { if } T_{1} \leq T_{2} \\ 0.8, & \text { if } T_{2}<T_{1}<5 * T_{2} \\ 1-T_{2} / T_{1}, & \text { if } T_{1} \geq 5 * T_{2} .\end{cases}
$$

The formula says that, given the number of distinct terms in C2 (i.e., $T_{2}$ ), the smaller the number of distinct terms in $\mathrm{C} 1$, $T_{1}$, is, the smaller the probability that a term in $C 2$ also appears in $\mathrm{C} 1$ will be; and, when $T_{1}$ becomes much larger than $T_{2}$, then $q$ will become closer to 1 ; otherwise, $q$ is 0.8 . Probability $p$ can be computed in a similar manner.

For parameters $\mathrm{B}$ (memory size) and $\alpha$, we assign a base value for each: $\mathrm{B}=10,000$ (pages) and $\alpha=5$. When the impact of a parameter is studied, we vary the values of the parameter while let the other parameter use its base value.

We present the following five groups of simulation results.

Group 1: In this group, a real collection will be used as both collection $\mathrm{C} 1$ and collection $\mathrm{C} 2$. Since there are three real collections (WSJ, FR, and DOE) and two parameters (B and $\alpha$ ), six simulation results will be collected. 
Group 2: In this group, different real collections will be used as $\mathrm{C} 1$ and $\mathrm{C} 2$. B will vary while $\alpha$ will use its base value. From the three real collections, six simulations can be designed.

Group 3: In this group, while $\mathrm{C} 1$ and $\mathrm{C} 2$ will continue to use real collections, only a small number of documents in C2 will be used to participate in the join. These experiments are used to investigate the impact of local selections. All simulations in this group use only the base values of the two parameters. Since there are three real collections, three simulation results will be collected in this group.

Group 4: In this group, $\mathrm{C} 1$ again will continue to use real collections, but $\mathrm{C} 2$ will be collections with only a small number of documents. The difference between Group 3 and Group 4 is that the former uses a small number of documents (in C2) from an originally large collection C2 and the latter uses an originally small collection C2. This difference has the following impacts on the cost:

1) documents in $C 2$ need to be read in randomly by the former but can still be read in sequentially by the latter; and

2) the size of the inverted file and the size of the B+tree on collection $\mathrm{C} 2$ for the former are computed based on the original collection, not just the documents used.

This will have an impact on the cost of algorithm VVM. In our experiments, after a real collection is chosen to be $\mathrm{C} 1, \mathrm{C} 2$ will be derived from $\mathrm{C} 1$. Again, all simulations in this group use only the base values of the two parameters. Since there are three real collections, three simulation results will be collected in this group.

Group 5: In this group, both collection $\mathrm{C} 1$ and collection $\mathrm{C} 2$ will use new collections but they will remain to be identical. Each new collection is derived from a real collection by reducing the number of documents in the real collection and increasing the number of terms in each document in the real collection by the same factor such that the collection size remains to be the same. The simulations in this group are especially aimed at observing the behavior of algorithm VVM. Again, only the base values of the two parameters will be used and three simulation results will be collected in this group since there are three real collections.

For space consideration, the simulation results for the backward order approach will not be presented. Notice that the backward order approach makes a difference only when HHNL and HVNL are used (see the discussions in Section 4.1 and Section 5.2). Compared with the forward order, the backward order requires more memory space to store intermediate similarities. As a result, the backward order with outer collection A1 and inner collection A2 incurs a somewhat higher cost than the forward order with outer collection B1 and inner collection B2 when A1 and B1 are the same collection and A2 and B2 are the same collection.

For all the figures in this section, a value $\mathrm{k}$ on $\mathrm{y}$-axis is equivalent to $10^{k}$ sequential page I/Os. For Figs. 1, 3, and 4, each unit on $\mathrm{x}$-axis is equivalent to 10,000 pages.

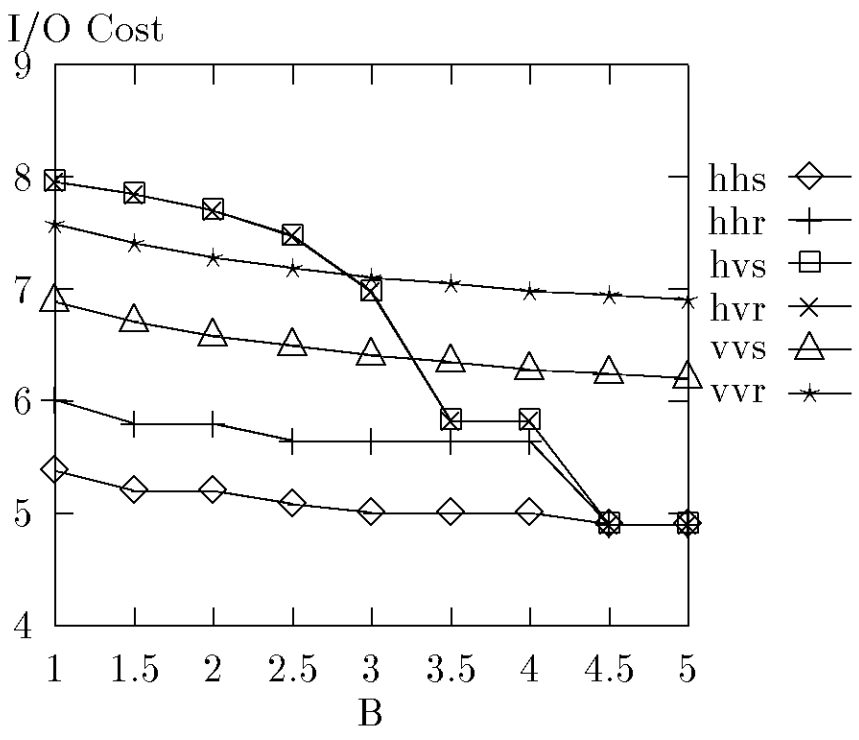

Fig. 1. Result of Simulation 1.

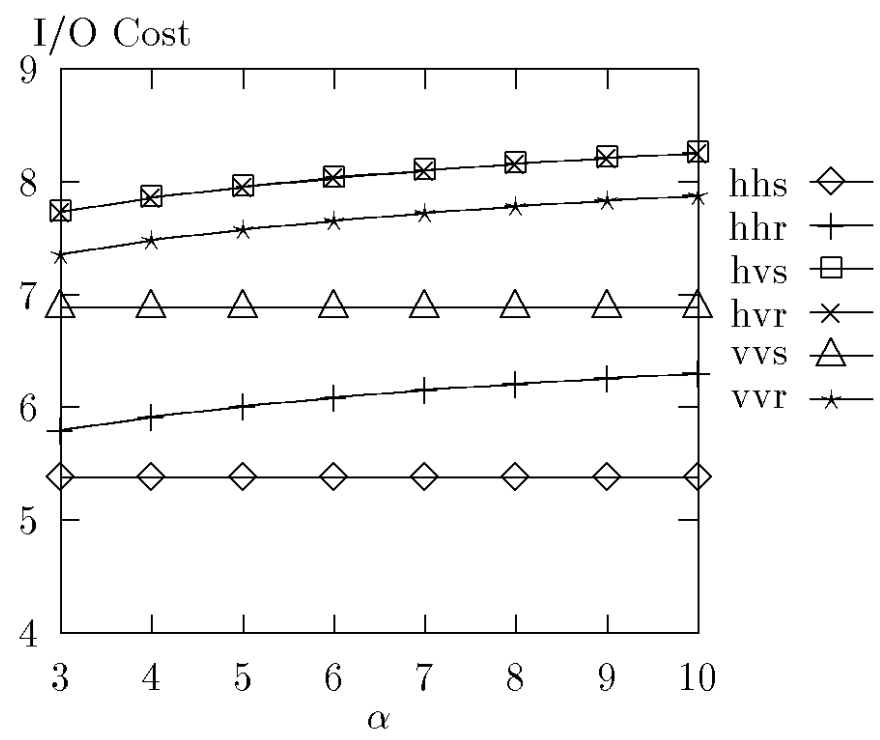

Fig. 2. Result of Simulation 4.

\section{Simulation Results in Group 1}

The following simulations are conducted in this group:

Simulation 1: $C 1=C 2=$ WSJ, $\alpha=5, B$ changes from 10,000 to 50,000 with an increment of 5,000

Simulation 2: $C 1=C 2=F R, \alpha=5, B$ changes from 10,000 to 50,000 with an increment of 5,000

Simulation 3: $C 1=C 2=D O E, \alpha=5, B$ changes from 10,000 to 50,000 with an increment of 5,000

Simulation 4: $C 1=C 2=W S J, B=10,000, \alpha$ changes from 3 to 10 with an increment of 1

Simulation 5: $C 1=C 2=F R, B=10,000, \alpha$ changes from 3 to 10 with an increment of 1

Simulation 6: $C 1=C 2=D O E, B=10,000, \alpha$ changes from 3 to 10 with an increment of 1

The following observations can be made from the result of simulation 1 (see Fig. 1). 
1) Algorithm HHNL outperforms the other two algorithms, especially when B is small.

2) There are several reasons that algorithm HVNL performs poorly. First, the outer document collection has too many documents $\left(N_{2}=98,736\right)$ which causes repeated read-ins of many inverted file entries on $\mathrm{C}$. Second, algorithm HVNL requires more random I/Os. Third, both $S_{2}$ and $J_{1}$ are not close to integers and, as a result, for each document or inverted file entry read in, algorithm HVNL incurs more than twice as much cost as that by algorithm HHNL (1 versus 0.41 for document and 1 versus 0.26 for inverted file entry).

3) The main reason that algorithm VVM performs very poorly is because the memory requirement for storing intermediate similarities (952,031 pages) is much greater than the available memory. As a result, many scans of the two inverted files are needed to process the join.

4) All algorithms perform better with larger available memory. When $B=45,000$ or larger, one document collection or an inverted file can be held in the memory in its entirety. When this happens, algorithm HHNL and algorithm HVNL have very similar performances since, in this case, algorithm HHNL scans each of the two document collections once and algorithm HVNL scans one document collection and one inverted file which has the same size as a document collection.

Similar observations as made from the result of simulation 1 can also be made from the results of Simulation 2 and Simulation 3 (not shown). Relatively speaking, the performance of algorithm VVM in Simulation 2 has the largest improvement due to the larger size of documents and fewer number of documents. However, the memory requirement for storing intermediate similarities in this case $(67,071$ pages) is still too large for the available memory to handle and at least two scans of the two inverted files are used to process the join. Not surprisingly, the relative performance of algorithm VVM in Simulation 3 has become much worse due to the smaller size of documents and larger number of documents.

The following observations can be made from the result of Simulation 4.

1) Algorithm HHNL is the best performer among the three algorithms.

2) hhs and vvs are independent of $\alpha$ because they involve no random I/Os.

3) Others become worse when $\alpha$ increases.

4) Algorithm HVNL is more sensitive to larger $\alpha$.

Similar observations can be made from the results of Simulations 5 and 6.

\section{Simulation Results in Group 2}

In this group, different real collections will be used as $\mathrm{C} 1$ and $\mathrm{C} 2$ and the base value for $\alpha$ will be used while B will vary. From the three real collections, the following six simulations can be designed.
Simulation 7: $C 1=W S J, C 2=F R, \alpha=5, B$ changes from 10,000 to 50,000 with an increment of 5,000

Simulation 8: C1 $=F R, C 2=$ WSJ $, \alpha=5, B$ changes from 10,000 to 50,000 with an increment of 5,000

Simulation 9: $C 1=F R, C 2=D O E, \alpha=5, B$ changes from 10,000 to 50,000 with an increment of 5,000

Simulation 10: $C 1=D O E, C 2=F R, \alpha=5, B$ changes from 10,000 to 50,000 with an increment of 5,000

Simulation 11: $C 1=W S J, C 2=D O E, \alpha=5, B$ changes from 10,000 to 50,000 with an increment of 5,000

Simulation 12: $C 1=D O E, C 2=W S J, \alpha=5, B$ changes from 10,000 to 50,000 with an increment of 5,000

Comparing the result of Simulation 7 with the result of Simulation 8 (see Figs. 3 and 4), the following observations can be made.

1) While algorithm HHNL is the best performer in Simulation 7, algorithm HVNL sometimes beats HHNL in Simulation 8. The reason is that, while algorithm HHNL lets the outer collection use as much memory space as possible, algorithm HVNL lets the inner collection use as much memory space as possible. For example, consider Fig. 4 when $B=35,000$ (i.e., $B=3.5$ in the figure). In this case, the entire inverted file on FR can be held in the memory. As a result, when algorithm HVNL is used, only one scan of WSJ and the inverted file on FR is needed to process the join. However, when algorithm HHNL is used, the memory is not large enough to hold the entire outer collection WSJ. As a result, one scan of WSJ and two scans of the inverted file on FR are needed to process the join when algorithm HHNL is used.

2) There is no change on the cost of algorithm VVM because it is completely symmetric to the two document collections.

3) When none of the two collections can be entirely held in the memory, we get mixed results for algorithm HHNL, that is, sometimes, it has a better result in Simulation 7 than that in Simulation 8, but sometimes the opposite is true. When only the smaller collection can be held in the memory, better performance can be achieved using the smaller collection as the outer collection. This is the reason that algorithm HHNL has a better result in Simulation 7 than that in Simulation 8 when B becomes 35,000 or larger. This observation also supports our earlier argument in Section 4.1 that the backward order can outperform the forward order if the backward order implies a much smaller outer collection.

4) The situation for algorithm HHNL is reversed for algorithm HVNL. The reason is that while algorithm HHNL lets the outer collection use as much memory space as possible, algorithm HVNL lets the inner collection use as much memory space as possible.

Similar observations made above between the result of Simulation 7 and the result of Simulation 8 can also be made between the result of Simulation 9 and the result of Simulation 10, as well as between the result of Simulation 11 and the result of Simulation 12 (the results of Simulations 912 are not shown). 


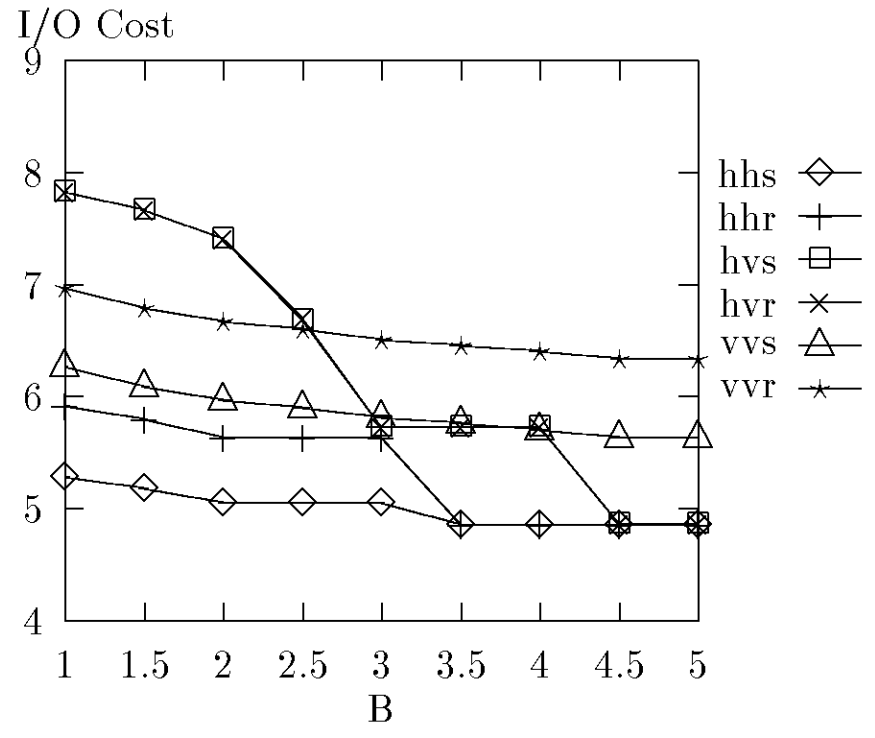

Fig. 3. Result of Simulation 7.

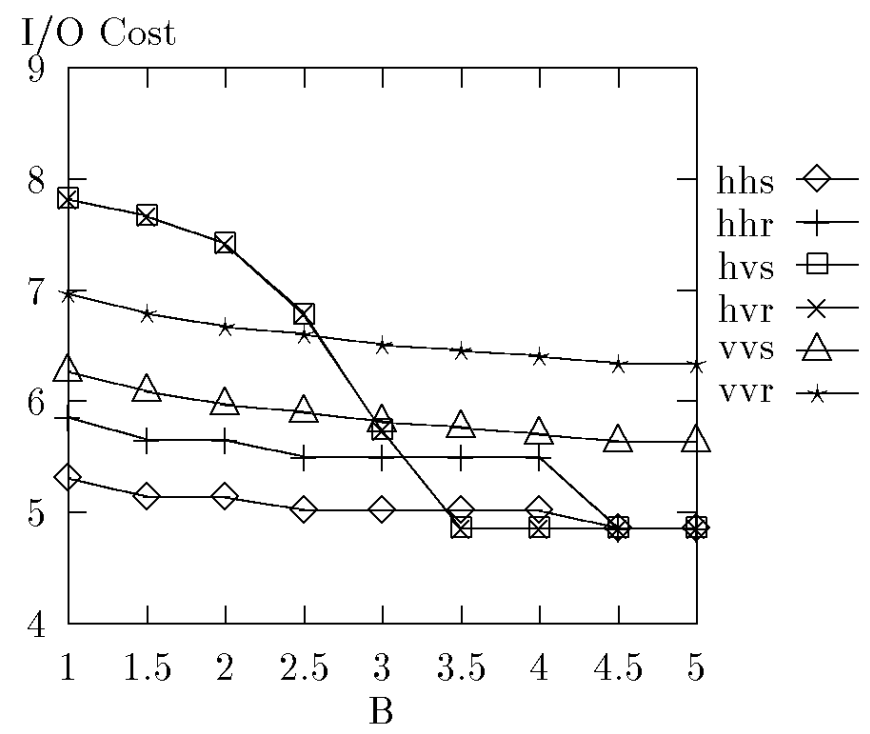

Fig. 4. Result of Simulation 8.

\section{Simulation Results in Group 3}

In this group, $\mathrm{C} 1$ and $\mathrm{C} 2$ will continue to be real collections but only a small number of documents in $\mathrm{C} 2$ will be used to participate in the join. Let $\mathrm{M}$ be the number of such documents in C2. Since $M \ll N_{2}$, we should read each of the M documents individually in random order. As a result, the cost of reading in the $\mathrm{M}$ documents will be $M *\left\lceil S_{2}\right\rceil * \alpha$. Based on this, we have the following new formula for $h h s$ :

$$
\text { hhs }=M *\left\lceil S_{2}\right\rceil * \alpha+\lceil(M / X) * D 1\rceil
$$

Since $\mathrm{M}$ is small, it is likely that all of the $\mathrm{M}$ documents in $\mathrm{C} 2$ can be held in the memory. In addition, the remaining memory space $\left((X-M) * S_{2}\right)$ can be used to read in as many documents in $\mathrm{C} 1$ as possible. As a result, we have the following formula for $h h r$ :

$$
h h r=h h s+\left\lceil D_{1} /\left((X-M) * S_{2}\right)\right\rceil *(\alpha-1)
$$

To compute hvs and hvr, we need to estimate the number of distinct terms in the $\mathrm{M}$ documents. This number can be estimated by $f(M)=T_{2}-\left(1-K_{2} / T_{2}\right)^{M} * T_{2}$. The cost formula for hos is the same as that in Section 5.2 except $D_{2}$ is replaced by $M *\left\lceil S_{2}\right\rceil * \alpha$ and $T_{2}$ is replaced by $f(M)$. Let this new formula be denoted by (HVS2). Since all I/Os in hos have become random I/Os, hor $=$ hvs.

The cost formulas for vos and vor remain the same. However, the memory requirement for storing the intermediate similarities is now reduced to $4 * N_{1} * M * \delta / P$. Other quantities such as the size of inverted file entries and the size of the B+tree on collection C2 remain as before.

The following three simulations are carried out:

Simulation 13: $C 1=C 2=W S J, B=10,000, \alpha=5, M$ changes from 5 to 50 with an increment of 5

Simulation 14: $C 1=C 2=F R, B=10,000, \alpha=5, M$ changes from 5 to 50 with an increment of 5

Simulation 15: $C 1=C 2=D O E, B=10,000, \alpha=5, M$ changes from 5 to 50 with an increment of 5

The following observations can be obtained from the result of Simulation 13 (see Fig. 5).

1) When $M$ is very small $(\leq 30)$, algorithm HVNL outperforms others as expected. Algorithm HHNL becomes the best performer when $\mathrm{M}$ becomes larger.

2) Since $M$ is so small, the $M$ documents can easily fit into the memory. As a result, algorithm HHNL requires only one scan of the inner document collection in addition to reading in the $\mathrm{M}$ documents from the outer collection.

3) In this case, the memory is able to accommodate all intermediate similarities for algorithm VVM. The reason that algorithm VVM incurs much higher cost than algorithm HHNL is because the size of the inverted file on collection $\mathrm{C} 2$ did not change although only a small number of documents in C2 is used.

Comparing the result of Simulation 14 (not shown) with the result of Simulation 13, a noticeable difference is that the relative performance of algorithm HVNL deterioratedalgorithm HVNL becomes worse than algorithm HHNL before $M$ reaches 10. This is because each document in FR contains much more terms than each document in WSJ and, therefore, more inverted file entries need to be read in by algorithm HVNL for processing a document in FR.

Comparing the result of Simulation 15 (not shown) with the result of Simulation 13, a noticeable difference is that the relative performance of algorithm HVNL is improvedalgorithm HVNL outperforms algorithm HHNL even after $\mathrm{M}$ reaches 50 . This is because each document in DOE contains much fewer terms than each document in WSJ and, therefore, fewer inverted file entries need to be read in by algorithm HVNL for processing a document in DOE.

For space considerations, we do not present simulation results for situations when the numbers of documents in both collections are reduced by selections. However, it is not difficult to see that comparing the situation when only one collection is reduced, algorithm HHNL will benefit the most when both collections are reduced. 


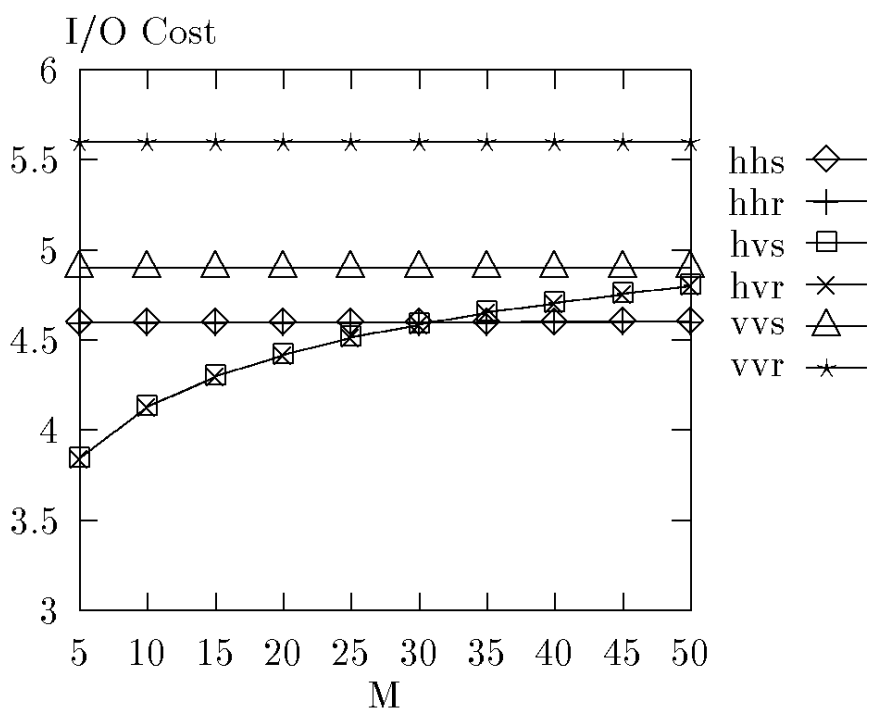

Fig. 5. Result of Simulation 13.

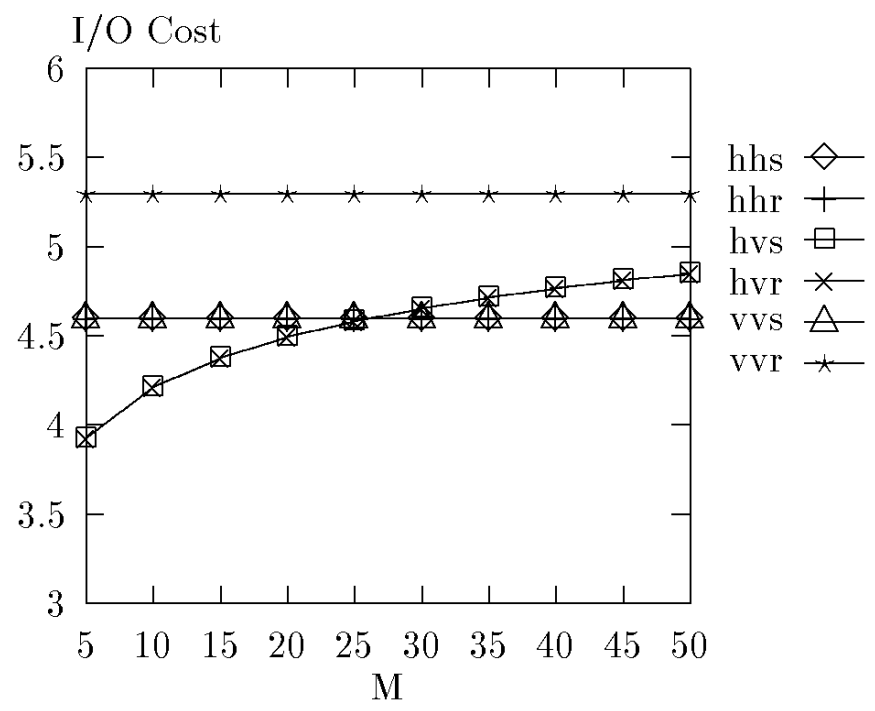

Fig. 6. Result of Simulation 16.

\section{Simulation Results in Group 4}

In this group, $\mathrm{C} 1$ will continue to use real collections, but C2 will be collections with only a small number of documents. Since we do not have real collections that contain a small number of documents, we derive such a collection from a real collection. This turns out to be quite easy. From a given document collection, we first keep its document size and then decide the number of documents we want in the new collection. From this number, say $M$, the number of distinct terms in the new collection can be computed by $f(M)$. Now, all key statistics of the new collection become available. With these statistics, the cost formulas in Section 5 can be used to find the cost of each algorithm.

The following three simulations are conducted in the group:

Simulation 16: $C 1=C 2=W S J, B=10,000, \alpha=5, M$ changes from 5 to 50 with an increment of 5
Simulation 17: $C 1=C 2=F R, B=10,000, \alpha=5, M$ changes from 5 to 50 with an increment of 5

Simulation 18: $C 1=C 2=D O E, B=10,000, \alpha=5, M$ changes from 5 to 40 with an increment of 5

Comparing the result of Simulation 16 (see Fig. 6) with that of Simulation 13 (see Fig. 5), the following observations can be made.

1) There is little change for algorithm HHNL. Since $M$ is so small, reading in the $\mathrm{M}$ documents sequentially or randomly makes little difference.

2) Algorithm HVNL degraded somewhat. This is the effect of $q$-the probability that a term in collection C2 also appears in collection C1. In Simulation 13, $q$ is computed based on the original $T_{1}$ and $T_{2}$. Since $T_{1}=$ $T_{2}, q=0.8$ is computed. In Simulation 16, $q$ is computed based on the original $T_{1}$ and the new $f(M)$. Since $f(M)$ is much smaller than $T_{1}, q$ between 0.92 to 0.99 are computed using the formula. Higher $q$ values imply that more inverted file entries on collection C1 need to be read in and as a result, the performance of algorithm HVNL is down.

3) The cost of algorithm VVM is reduced substantially. The main reason behind the reduction is the reduction of the size of the inverted file on C2. In Simulation 13, the size is computed based on the original $\mathrm{C} 2$, but, in Simulation 16, the size is computed based on the reduced collection.

Similar observations as above can be made for Simulation 17 and Simulation 18.

\section{Simulation Results in Group 5}

In this group, both $\mathrm{C} 1$ and $\mathrm{C} 2$ will use new collections but they will remain to be identical. Each new collection is derived from a real collection by reducing the number of documents and increasing the number of terms in each document in the real collection by the same factor $\mathrm{F}$ to ensure that the collection size remains to be the same.

The following three simulations are carried out:

Simulation 19: $C 1=C 2$ are derived from WSJ, $B=10,000, \alpha=5$, the decreasing (increasing) factor changes from 1 to 13 with an increment of 2

Simulation 20: $C 1=C 2$ are derived from $F R, B=10,000, \alpha=5$, the decreasing (increasing) factor changes from 1 to 5 with an increment of 1

Simulation 21: $C 1=C 2$ are derived from $D O E, B=10,000, \alpha=5$, the decreasing (increasing) factor changes from 1 to 28 with an increment of 3

The following observations can be made from the result of Simulation 19 (see Fig. 7).

1) When factor $F$ is small $(\leq 5)$, algorithm HHNL outperforms other algorithms. However, when F is 7 or larger, the sequential version of algorithm VVM (i.e., vvs) becomes the best performer.

2) vos decreases rapidly as $\mathrm{F}$ increases as expected. When $F$ reaches 11, all intermediate similarities can be held in the memory. As a result, vvs reaches its lower bound-each inverted file is scanned once. When $\mathrm{F}=$ 11 , the number of documents in the collection is 


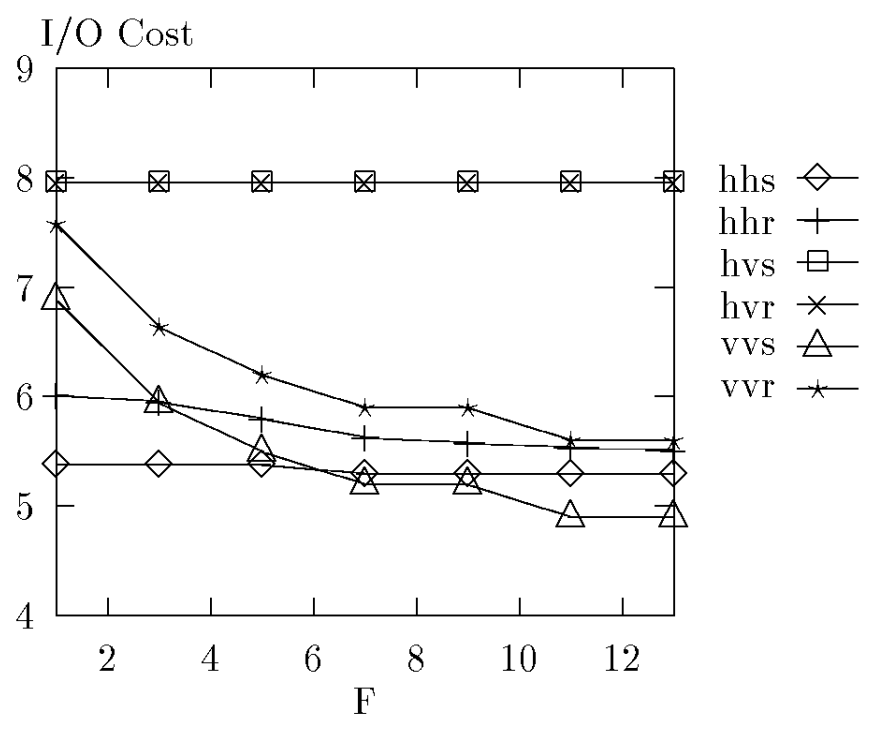

Fig. 7. Result of Simulation 19.

reduced to 8,976 and the number of terms in each document becomes 3,619.

3) hos and hor are insensitive to the changes.

4) $h h r$ decreases as F increases. This is because as F increases, the number of documents in $\mathrm{C} 1$ decreases. Since the number of random I/Os is bounded by the number of documents in $\mathrm{C} 1, h \mathrm{hr}$ decreases as a result.

Similar observations as for Simulation 19 can be made for Simulation 20 with the only difference that vos reaches its minimum faster for the latter. The reason is that the number of documents in FR is originally much smaller than that in WSJ. Again, similar observations as for Simulation 19 can be made for Simulation 21 with the only difference that vos reaches its minimum slower for the latter. The reason is that the number of documents in DOE is originally much larger than that in WSJ.

\subsection{Summary of the Simulation Results}

The following main points can be summarized from the above extensive simulations.

1) The cost of one algorithm under one situation can differ drastically from that of another algorithm under the same situation. For example, in Simulation 1, Algorithm HVNL incurs a cost which is about 4,000 times higher than that of Algorithm HHNL when the memory buffer is small ( $\mathrm{B}=10,000)$; but, in Simulation 13, the cost incurred by Algorithm HHNL is more than five times higher than that by Algorithm HVNL. As a result, it is important to choose an appropriate algorithm for a given situation.

2) If the number of documents in one of the two document collections, say $M$, is originally very small or becomes very small after a selection, then algorithm HVNL has a very good chance to outperform other algorithms. Although how small $\mathrm{M}$ needs to be to be small enough mainly depends on the number of terms in each document in the outer collection, $\mathrm{M}$ is likely to be limited by 100 (it is 70 for Simulation 15).
3) If the number of documents in each of the two collections is not very large (roughly $N_{1} * N_{2}<10,000 * B$ ) and both document collections are large such that none can be entirely held in the memory, then algorithm VVM (the sequential version) can outperform other algorithms.

4) For most other cases, the simple algorithm HHNL performs very well.

5) The costs of the random versions of these algorithms depict the worst case scenario when the I/O devices are busy satisfying different obligations at the same time. Except for algorithm VVM, these costs have no impact in ranking these algorithms.

Overall, the simulation results match well with our analysis in Section 5.4.

\subsection{An Integrated Algorithm}

Since no one algorithm is definitely better than all other algorithms in all circumstances, it is desirable to construct an integrated algorithm that can automatically determine which algorithm to use given the statistics of the two collections $\left(N_{1}, N_{2}, K_{1}, K_{2}, T_{1}, T_{2}, p, q, \delta\right)$, system parameters $(B, P, \alpha)$ and query parameters $(\lambda$, selectivities of predicates on nontextual attributes). This integrated algorithm can be sketched as follows:

If none of the two collections has inverted file $/^{*}$ in this case, only HHNL can be used * /

\{compute hhs using formula (HHS1);

compute bhhs; /* the counterpart of hhs when the backward order is used (formula not shown) */

If $h h s \leq b h h s$, use the forward order of HHNL;

Else use the backward order of HHNL;

\}

If only one collection has inverted file /* only HHNL and HVNL can be used in this case * /

\{If there is no selection

\{compute hhs using formula (HHS1);

compute bhhs;

compute hos using formula (HVS1);

compute bhvs; $/^{*}$ the counterpart of hvs when the

backward order is used (formula not shown) */

$$
\}
$$

Else

\{estimate the number of documents that can partici-

pate in the join using the selectivities;

compute hhs using formula (HHS2);

compute bhhs;

compute hos using formula (HVS2);

compute bhos;

$$
\text { \} }
$$

use the algorithm with the lowest estimated cost; \}

If both collections have inverted file

\{If there is no selection

\{compute hhs using formula (HHS1);

compute bhhs;

compute hos using formula (HVS1);

compute bhos; /* the counterpart of hos when the backward order is used (formula not shown) */ 
compute vvs using formula (VVS);

$$
\}
$$

\section{Else}

\{estimate the number of documents that can participate in the join using the selectivities;

compute hhs using formula (HHS2);

compute bhhs;

compute hos using formula (HVS2);

compute bhos;

compute vos using formula (VVS);

\}

use the algorithm with the lowest estimated cost; \}

\section{Concluding Remarks}

In this paper, we presented and analyzed three algorithms for processing joins between attributes of textual type. From analysis and simulation, we identified, for each algorithm, the type of input document collections with which the algorithm is likely to perform well. More specifically, we found that algorithm HVNL can be very competitive only when the number of documents in one of the two document collections is/becomes very small, and algorithm VVM can perform very well when the number of documents in each of the two collections is not very large and both document collections are large such that none can be entirely held in the memory. In other cases, algorithm HHNL is likely to be the top performer. Since no one algorithm is definitely better than all other algorithms, we proposed the idea of constructing an integrated algorithm consisting of the basic algorithms such that a particular basic algorithm is invoked if it has the lowest estimated cost. We also indicated that the standardization of term numbers will be very useful in multidatabase environments.

Further studies in this area include:

1) investigate the impact of the availability of clusters on the performance of each algorithm;

2) develop cost formulas that include CPU cost and communication cost;

3) develop algorithms that process textual joins in parallel; and

4) conduct more detailed simulation and experiment.

\section{ACKNOWLEDGMENTS}

We thank the anonymous reviewers for their valuable suggestions to improve the paper. This research is supported in part by the following grants: U.S. National Science Foundation grants under IRI-9309225 and IRI-9509253, U.S. Air Force under AFOSR 93-1-0059, NASA under NAGW-4080, and ARO under BMDO grant DAAH04-0024.

\section{REFERENCES}

[1] D. Beech, P. Chellone, and C. Ellis, "An ADT Approach to Full Text," ISO/IEC JTC1/SC21/WG3 DBL CBR-57, 1992.

[2] P. Bernstein, E. Wong, C. Reeve, and J. Rothnie, "Query Processing in a System for Distributed Databases (SDD-1)," ACM Trans. Database Systems, vol. 6, no. 4, pp. 602-625, Dec. 1981.
[3] C. Buckley, G. Salton, and J. Allan, "Automatic Retrieval with Locality Information Using Smart," Proc. First Text Retrieval Conf., pp. 59-72, Gaithersburg, Md., Mar. 1993.

[4] U. Dayal, and H-Y. Hwang, "View Definition and Generalization for Database Integration in a Multidatabase system," IEEE Trans. Software Eng., vol. 10, no. 6, pp. 628-644, Nov. 1984.

[5] W. Du, R. Krishnamurthy, and M. Shan, "Query Optimization in Heterogeneous Databases," Proc. VLDB Conf., pp. 277-291, Vancouver, B.C., Canada, Aug. 1992.

[6] S. Dumais, and J. Nielson, "Automating the Assignment of Submitted Manuscripts to Reviewers," Proc. ACM SIGIR Conf., Copenhagen, June 1992.

[7] S. Ghose, "File Organization: The Consecutive Retrieval Property," Comm. ACM, vol. 15, no. 9, pp. 802-808, Sept. 1972.

[8] D. Harman, "Overview of the First Text Retrieval Conference," D. Harman, ed., Computer Systems Technology, U.S. Dept. of Commerce, National Institute of Statistics \& Technology, 1993.

[9] W. Litwin, L. Mark, and N. Roussopoulis, "Interoperability of Multiple Autonomous Databases," ACM Computing Surveys, vol. 22, no. 3, pp. 267-293, Sept. 1990.

[10] C. Liu, Introduction to Combinatorial Mathematics. McGraw-Hill, 1968.

[11] L. Lilian and B. Bhargava, "A Scheme for Batch Verification of Integrity Assertions in a Database System," IEEE Trans. Software Eng., vol. 10, no. 6, pp. 664-680, Nov. 1984.

[12] W. Meng and C. Yu, "Query Processing in Multidatabase Systems," Modern Database Systems: The Object Model, Interoperability, and Beyond, W. Kim, ed., chapter 27, pp. 551-572. AddisonWesley/ACM Press, 1995.

[13] W. Meng, C. Yu, and W. Kim, "A Theory of Translation from Relational Queries to Hierarchical Queries," IEEE Trans. Knowledge and Data Eng., vol. 7, no. 2, pp. 228-245, Apr. 1995.

[14] G. Salton and M. McGill, Introduction to Modern Information Retrieval. McGraw-Hill, 1983.

[15] L. Saxton and V. Raghavan, "Design of an Integrated Information Retrieval/Database Management System," IEEE Trans. Knowledge and Data Eng., vol. 2, no. 2, pp. 210-219, June 1990.

[16] A. Sheth and J. Larson, "Federated Database Systems for Managing Distributed, Heterogeneous, and Autonomous Databases," ACM Computing Surveys, vol. 22, no. 3, pp. 183-236, Sept. 1990.

[17] R. Swaminathan and D. Wagner, "On the Consecutive-Retrieval Problem," SIAM J. Computing, vol. 23, no. 2, pp. 398-414, Apr. 1994.

[18] A. Tomasic, H. Garcia-Molina, and K. Shoens, "Incremental Updates of Inverted Lists for Text Document Retrieval," Proc. ACM SIGMOD Conf., pp. 289-300, Minneapolis, May 1994.

[19] C. Yu, Y. Zhang, W. Meng, W. Kim, G. Wang, T. Pham, and S. Dao, "Translation of Object-Oriented Queries to Relational Queries," Proc. IEEE Conf. Data Eng., pp. 90-97, Taipei, Taiwan, Mar. 1995.

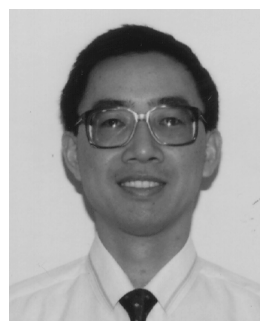

Weiyi Meng (M'93) received the BS degree in mathematics from Sichuan University, Chengdu, People's Republic of China, in 1982, and the MS and $\mathrm{PhD}$ degrees in electrical engineering and computer science from the University of Illinois at Chicago in 1988 and 1992, respectively. $\mathrm{He}$ is currently an assistant professor in the Department of Computer Science at the State University of New York at Binghamton. His research interests include Internet-based information retrieval, multidatabase systems, query processing, and optimization. He is a member of the IEEE. 


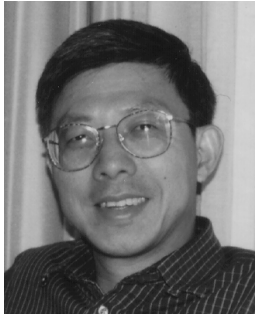

Clement Yu obtained his BSc degree from Columbia University in 1970 and his PhD degree from Cornell University in 1973. Currently, he is a professor in the Department of Electrical Engineering and Computer Science at the University of Illinois at Chicago. His research areas are database and information (multimedia) retrieval. He has served as an advisory committee member for the U.S. National Science Foundation and as program chair and general chair for several national and international conferences and workshops. He is currently an editorial member/associate editor for IEEE Transactions on Knowledge and Data Engineering, Distributed and Parallel Databases, and the International Journal of Software Engineering and Knowledge Engineering. His publications appear in leading ACM and IEEE journals/transactions, as well as in major conference proceedings. He is a senior member of the IEEE.

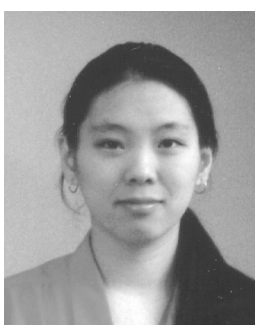

Wei Wang received her MS degree in system science in 1995 from the State University of New York at Binghamton. She is currently a PhD student in computer science at the University of California, Los Angeles. She has coauthored 12 papers in the area of database and imprecise reasoning. Her current research areas include spatial database systems and data mining.

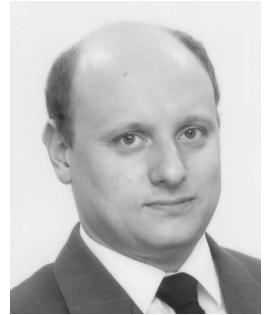

Naphtali Rishe completed his PhD degree at Tel Aviv University in 1984. His expertise is in database management. His methodology for the design of database applications and his work on the Semantic Binary Database Model were published as a book by Prentice Hall in 1988. Dr. Rishe's Semantic Modeling theory was published as a book by McGraw-Hill in 1992. His current research focuses on efficiency and flexibility of database systems (particularly of object-oriented, semantic, decision-support, and spatial/geographic DBMS), distributed DBMS, high-performance systems, database design tools, and Internet access to databases. He is the editor of three books and author of 23 papers in journals, seven chapters in books and serials (including three in Springer-Verlag's Lecture Notes in Computer Science), and more than 50 papers in proceedings. Dr. Rishe has been awarded millions of dollars in research grants by government and industry. His research is currently sponsored by NASA, NATO, BMDO, ARO, DoD, Dol, and other agencies. Dr. Rishe also has extensive experience in database applications and database systems in the industry. This includes eight years of employment as head of software and database projects (1976-1984) and, later, consulting for companies such as Hewlett-Packard and the telecommunications industry. Since receiving his $\mathrm{PhD}$, he has worked as an assistant professor at the University of California, Santa Barbara (1984-1987), and associate professor (1987-1992) and professor (1992-present) at Florida International University (FIU). Dr. Rishe is the founder and director of the High Performance Database Research Center at FIU, which now employs 85 researchers. Dr. Rishe chaired the program and steering committees of the PARBASE conference and is on the steering committee of the PDIS conference series. He is a member of the IEEE Computer Society. 